\title{
Synthesis of aligned single walled nanotubes using catalysts defined by nanosphere lithography
}

Koungmin Ryu, Alexander Badmaev, Lewis Gomez, Fumiaki Ishikawa, Bo Lei and Chongwu Zhou*

Department of Electrical Engineering, University of Southern California,

Los Angeles, California 90089.

\section{Supporting Information}

\section{Supporting Information Available:}

S1. Detailed calculation of the geometrical factor $S(\alpha, \varphi)$ for packed monolayer and bilayer nanospheres; S2. Detailed calculation of the catalyst nanoparticle size with different deposition angles and different packed nanosphere structures;

S3. AFM image and diameter distribution of catalyst arrays prepared using packed bilayers of $100 \mathrm{~nm}$ nanospheres;

S4: Raman spectrum of nanotubes showing negligible D-band and RBM mode.

\section{Detailed calculation of the geometrical factor $S(\alpha, \varphi)$ for packed monolayer and bilayer nanospheres}

Geometrical factor $\boldsymbol{S}(\boldsymbol{\alpha}, \boldsymbol{\varphi})$ equals to the area of opening between 3 adjacent spheres for a closely packed monolayer (or 6 for a bilayer) of unit radius as projected onto a plane perpendicular to the deposition direction, where $\boldsymbol{\alpha}$ is deposition angle, which is the angle between the deposition direction and normal of the substrate plane, and $\boldsymbol{\varphi}$ is azimuthal angle, which is the angle between the projection of deposition direction to the substrate plane and orientation of the closely packed lattice of spheres. Numerical integration is done using Monte-Carlo method.
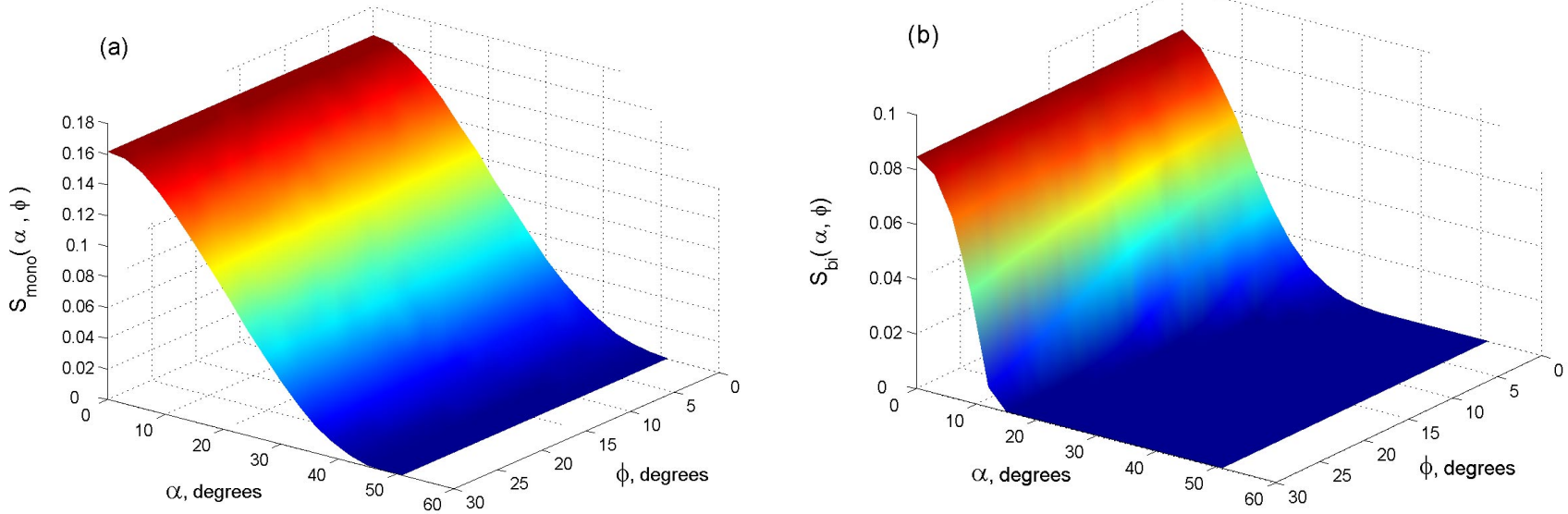

Figure S1. Calculated geometrical factor $\boldsymbol{S}(\alpha, \varphi)$ for a monolayer (a) and for a bilayer of packed nanospheres. 


\section{Detailed calculation of the catalyst nanoparticle size with different deposition angles and different packed nanosphere structures}

Based on the geometrical factor $\boldsymbol{S}(\boldsymbol{\alpha}, \boldsymbol{\varphi})$, we have calculated the catalyst particle diameter for relevant sizes of nanospheres. Figure S2 shows the expected diameter of the annealed catalysts prepared using 6 different nanosphere structures (monolayers and bilayers of $200 \mathrm{~nm}, 100 \mathrm{~nm}$ and $50 \mathrm{~nm}$ nanospheres) as a function of the deposition angle ( $\boldsymbol{\alpha})$ with $5 \AA$ metal film deposited, where the azimuthal angle $(\boldsymbol{\varphi})$ is fixed at zero degree. For nanosphere bilayers, as the deposition angle ( $\boldsymbol{\alpha}$ ) approaches $35^{\circ}$, the catalyst size goes to zero, while the catalyst size for nanosphere monolayers goes to zero around $56^{\circ}$ of deposition angle.

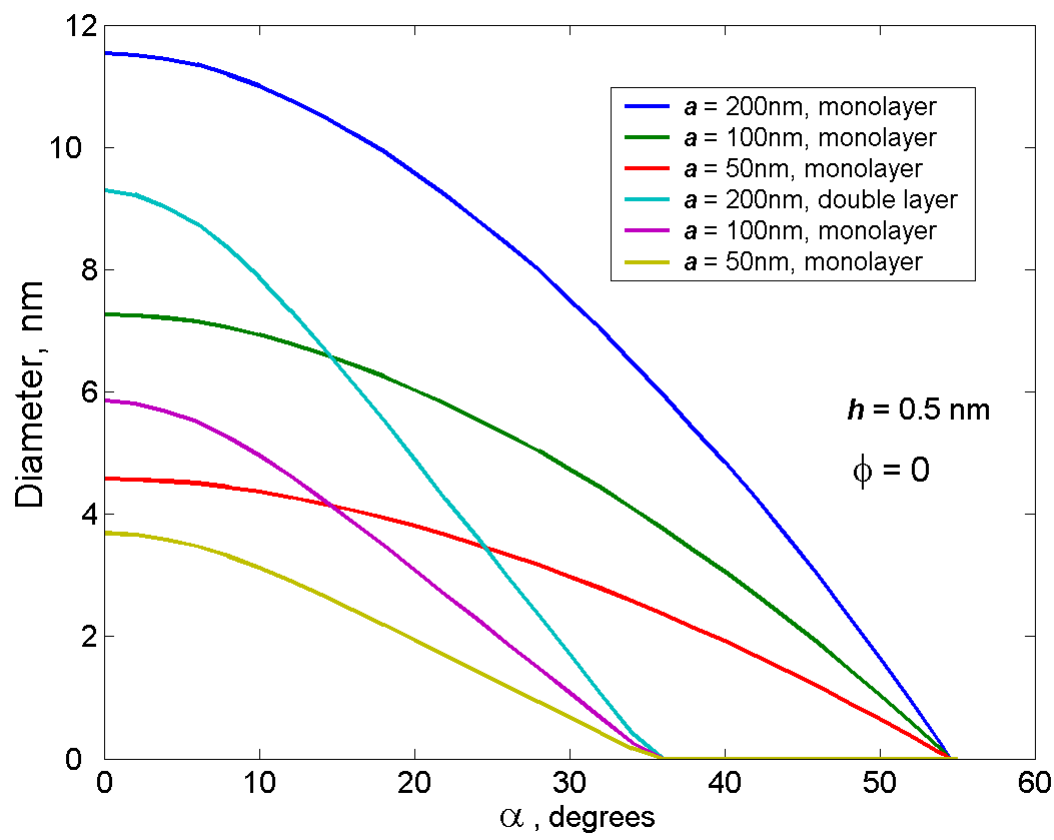

Figure S2. Calculated catalyst nanoparticle diameter v.s. the metal deposition angle for monolayers and bilayers of 200 $\mathrm{nm}, 100 \mathrm{~nm}$ and $50 \mathrm{~nm}$ nanospheres. 


\section{AFM image and diameter distribution of catalyst arrays prepared using packed bilayers of $100 \mathrm{~nm}$ nanospheres}

The catalyst arrays were prepared using nanosphere lithography with a packed bilayer of $100 \mathrm{~nm}$ nanospheres, followed by $20 \AA$ Fe deposition and annealing at $900{ }^{\circ} \mathrm{C}$ for $10 \mathrm{~min}$. The catalyst array exhibits a triangular lattice as shown in Figure S3 (a). The Gaussian fit to the histogram gives a diameter distribution of $6.44 \pm 1.35 \mathrm{~nm}$ (Figure S3 (b)).
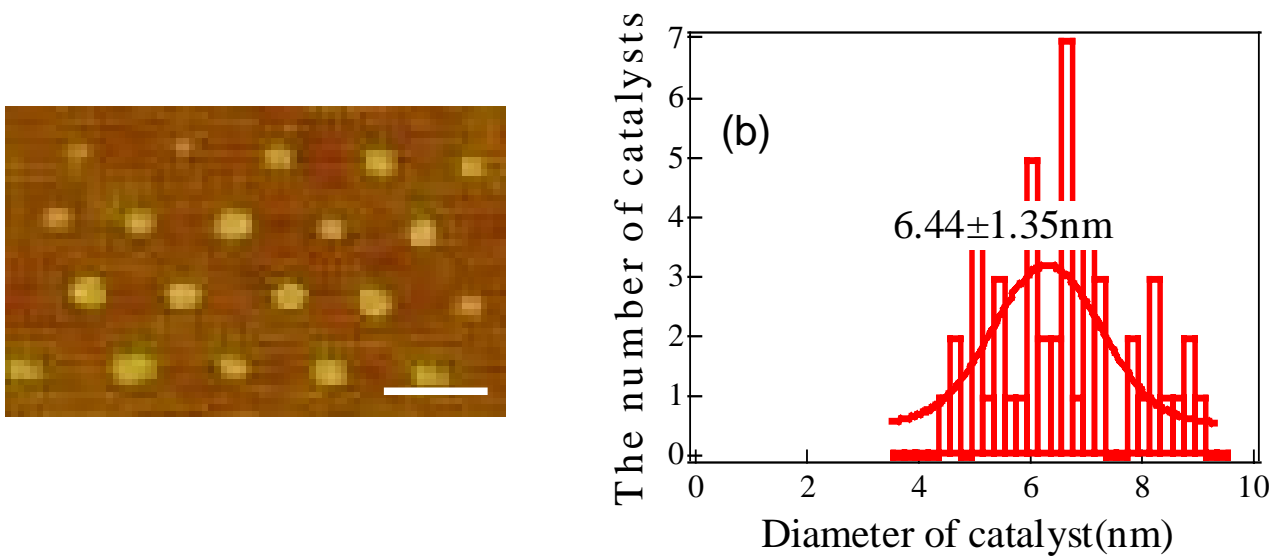

Figure S3. (a) AFM image of catalyst arrays prepared using nanosphere lithography with a packed bilayer of 100 $\mathrm{nm}$ nanospheres and with $20 \AA \mathrm{Fe}$ deposited. (b) Histogram of the diameter of catalyst nanoparticles.

\section{Raman spectrum of nanotubes showing negligible D-band and RBM mode}

Figure S4 (a) shows a representative Raman spectrum of nanotube exhibiting negligible D-band, confirming very clean and defect-free nanotubes were grown. Figure S4 (b) displays the RBM mode of a typical carbon nanotube prepared using nanosphere lithography. A single RBM peak at $175 \mathrm{~cm}^{-1}$ confirms that this nanotube is single-walled. Other peaks in Figure $S 4$ (b) are from the quartz substrate.
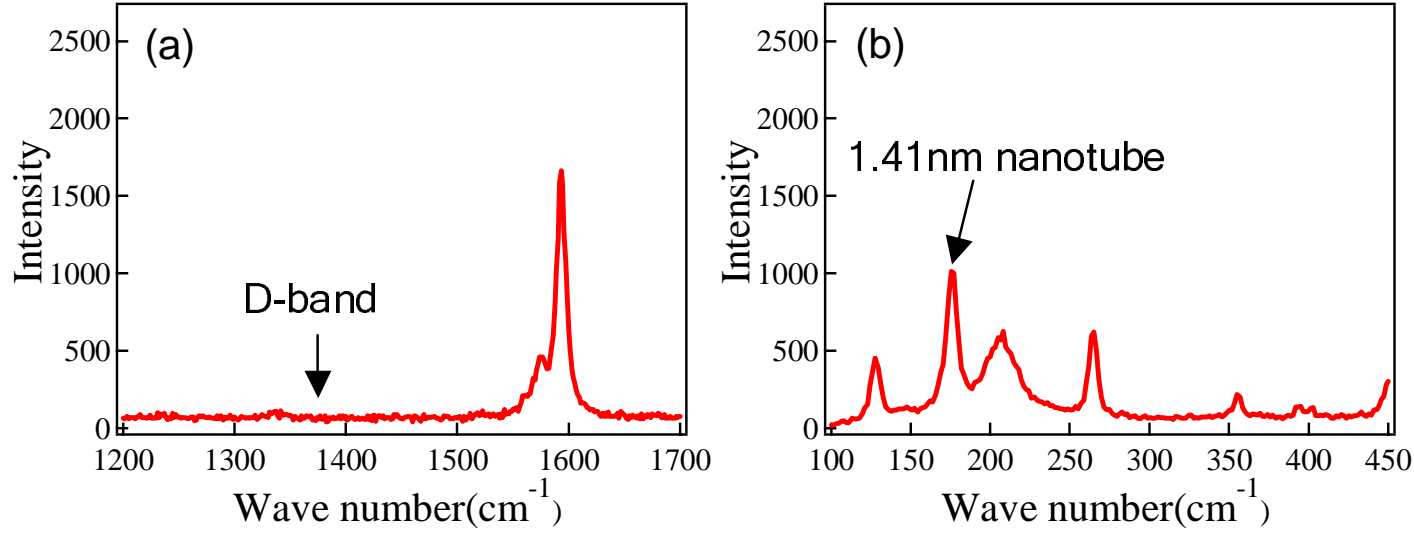

Figure S4. Raman spectrum of a nanotube. 
